\title{
Este es el fin de la escuela tal y como la conocemos. Unas reflexiones en tiempo de confinamiento
}

\author{
This is the End of the School as We Know it. \\ Some Reflections in Time of Confinement \\ Rafael Feito'
}

\begin{abstract}
Resumen
La crisis provocada por la COVID-19 ha situado a la escuela en un escenario inédito. De un día para otro, la enseñanza -cuando esto ha sido posible- ha pasado de ser presencial a serlo virtual. Se desconoce tanto el porcentaje de profesores como de alumnos que pueden estar enseñando y aprendiendo telemáticamente y a qué estrategias están recurriendo. En este escenario, las diferencias en términos de capital económico y cultural se agravan. Si no se adoptan medidas radicales, es posible que este periodo sin docencia presencial pueda pasar factura a toda una generación. Profesores, alumnos y padres y madres están accediendo a contenidos didácticos -del tipo de la academia Khanque ponen de manifiesto que es posible aprender de otra manera, de un modo más autónomo. Esto puede traducirse en una redefinición creativa e innovadora del papel del profesor. Es posible que, a partir de la vuelta a la docencia presencial, no sea preciso pasar tantas horas en la escuela y, muy especialmente, en el aula. La universidad, igualmente, debería aprovechar la ventaja de contar con más medios y con un estudiantado más conectado a la red que el del resto de los niveles educativos para acometer una transformación profunda de su docencia.
\end{abstract}

\section{Palabras clave}

Enseñanza virtual, innovación educativa, capital cultural, desigualdades educativas, enseñanza universitaria.

\section{Abstract}

The crisis caused by COVID-19 has placed the school in an unprecedented scenario. From one day to the next, teaching -when this has been possible- has moved from being face-to-face to being virtual. It remains unknown what percentage of teachers and students may be teaching and learning telematically and to what strategies they are resorting. Under these circumstances, the differences among students in terms of economic and cultural capital are aggravated. If radical measures are not taken, it is likely that this period without face-to-face teaching may take its toll on an entire generation. Teachers, students and parents are now accessing educational content -of the kind provided by the Khan Academy- which shows that it is possible to learn in a different autonomous way. This can lead to a creative and innovative redefinition of the role of the teacher. It is possible that, as of the return to face-to-face teaching, it will not be necessary to spend so many hours in the school and, very especially, in the classroom. The university, likewise, should take advantage of the fact that it has more resources and a student body that is more easily connected to the Internet than that of the rest of the educational levels, in order to undertake a deep transformation of its teaching.

\section{Keywords}

Online teaching, educational innovation, cultural capital, educational inequalities, university teaching.

\section{Cómo citar/Citation}

Feito, Rafael (2020). Este es el fin de la escuela tal y como la conocemos. Unas reflexiones en tiempo de confinamiento. Revista de Sociología de la Educación-RASE, 13 (2) Especial, COVID-19, 156-163. http://dx.doi.org/10.7203/RASE.13.2.17130. 


\section{Introducción}

La gravísima crisis que -en el momento de escribir estas líneas- está suponiendo la pandemia de la COVID-19 ha introducido un cambio radical en el modo de afrontar la docencia y el aprendizaje. El confinamiento obliga a trabajar telemáticamente. Es obvio que aún carecemos de información suficiente para saber cómo se está resolviendo este problema en los diferentes niveles educativos (desde la primaria hasta la universidad $)^{2}$. En la primaria y en la secundaria hay quejas - pero se desconoce su grado de representatividad- con relación al incremento desmesurado de los deberes (problema que ya existía antes de la pandemia). Grave es la situación de los estudiantes del último curso de bachiller, sobre los cuales pende la espada de Damocles de la lamentable prueba de acceso a la universidad. En los distintos niveles de la formación profesional y en ciertos grados y másteres universitarios, las prácticas se han visto interrumpidas y algunas simplemente no se realizarán, o quizás cabrá la posibilidad de recurrir al periodo estival para acometerlas.

Esta crisis está poniendo aún más de manifiesto la brecha que separa a los alumnos en función de sus capitales cultural y económico. Los padres -más bien madres- con altos niveles educativos se pueden convertir en tutores de sus hijos. Quienes gozan de mayor capacidad económica pueden contar con una buena conexión a Internet, varios ordenadores en el hogar y la posibilidad de contratar profesores particulares online. Una vez que salgamos de esta crisis, veremos un efecto similar al llamado verano del olvido: las largas vacaciones del verano acrecientan las desigualdades educativas entre unas clases sociales y otras.

Es posible que esta crisis sea un mero paréntesis tras el cual se volvería a trabajar en la escuela del mismo modo que anteriormente. Sin embargo, una situación tan excepcional como la actual podría ser una inigualable ocasión para mejorar transformando radicalmente la escuela, y esta es la razón por la que el título de este texto parafrasea el de una famosa canción de R.E.M.

\section{Una de cal y otra de arena}

Muchos tecnófilos se pueden estar frotando las manos ante el posible advenimiento de una nueva manera de trabajar en la escuela. Conviene, sin embargo, señalar algún que otro aviso para navegantes. Si bien es cierto que hay ya muchas aulas que cuentan con pantallas virtuales y centros cuyos alumnos utilizan tabletas, no está nada claro que en tales aulas y centros no se esté haciendo aproximadamente lo mismo que con un encerado tradicional, y que los libros de texto de las tabletas no vayan más allá de un mero cambio de formato - del papel a la pantalla-. Quiero decir con esto que las Tecnologías de la Información y de la Comunicación (TIC), pese a ser elementos que posibilitan el cambio, no alteran por sí solas las relaciones de poder dentro del aula ni las maneras de enseñar y de aprender. En una intervención publicada en estos días, un profesor decía lo siguiente: «No os equivoquéis, mandar los mismos ejercicios, pero por plataforma no es competencia digital» (Fórum Aragón, 2020: 24). Es más, las TIC podrían contribuir a adocenar al estudiantado o, simplemente, habituarle a no ir más allá de encontrar la respuesta correcta. Así, y a modo de ejemplo, en el caso de las Matemáticas de la ESO, cualquier alumno puede encontrar fácilmente la solución a los inevitables problemas de trenes que se van a encontrar en un punto determinado o de recipientes que se llenan de agua con varios grifos abiertos al tiempo que se vacían por un conducto. Se trata de problemas tan sumamente trillados que basta con escribir en Google «problemas sobre grifos» o las primeras palabras del enunciado del problema para que aparezcan decenas -cuando no centenares- de

2 Pese a que han salido a relucir preocupaciones sobre la lectoescritura, entiendo que no tendría mucho sentido preocuparse por el escaso avance en los contenidos curriculares de la educación infantil. 
páginas que permiten salir airoso de tales tareas. Una pizarra digital, por su parte, podría permitir relegar la clase a una sucesión de vídeos desconectados de las inquietudes del alumnado. Tendríamos, eso sí, una enseñanza más entretenida, pero no más interesante.

Quienes tienen hijos en edad escolar habrán tenido ocasión de comprobar las excelentes lecciones que se pueden encontrar en la red -sobre todo en youtube, y a cargo de profesores de muy distinto tipo- de una calidad muy superior a las habituales de un aula convencional. Buena parte de estos docentes -que en muchos casos no cuentan con una formación inicial como profesores- son personas que saben comunicar. Con el apoyo de un par de cámaras, una iluminación adecuada y, si es el caso, con paquetes informáticos, son capaces de conseguir que la gente aprenda. No siempre se recurre a medios particularmente sofisticados. Damián Pedraza, un profesor youtuber de Matemáticas, no va más allá de una pizarra y varias cámaras. Personalmente, me gusta que recurra a un encerado y no a una pizarra digital o a un programa de escritura en pantalla. De esta manera, el ritmo de la explicación se acerca a una secuenciación natural. Otros docentes, como David Calle, introducen fragmentos de películas en sus apasionantes explicaciones de Matemáticas y de Física ${ }^{3}$. Lo bueno de esta enorme cantidad de vídeos es que cada cual puede elegir el que más le convenga a su estilo de aprendizaje. No hay duda de que el profesor podría ejercer de asesor con respecto a qué videos utilizar. Si nos vamos al aprendizaje de idiomas -singularmente el inglés-, los recursos son simplemente inabarcables en variedad y en calidad ${ }^{4}$. Del mismo modo que se consideró que el libro de texto era una garantía frente al mal profesor, la red podría serlo frente al mal docente y al mal libro de texto.

¿Por qué digo que las TIC deberían ser claves para la promoción de un cambio en las relaciones de poder? Las TIC pueden posibilitar que el centro de gravedad de los procesos de enseñanza-aprendizaje se traslade de la persona que enseña a la persona que aprende. Algo de esto se está haciendo en algunos centros con la llamada clase invertida, término acuñado por los profesores de secundaria Jonathan Bergmann y Aaron Sams en su libro sobre esta alternativa escolar (2014)5. Muy resumidamente, la clase invertida consiste en que el alumnado aprende el contenido de cada lección o tema por medio de un vídeo seleccionado -o, como en el caso de estos docentes, preparado por ellos mismos- fuera del horario lectivo, de manera que en la sesión del día siguiente se aclaran los contenidos a partir de las dudas o sugerencias planteadas por los estudiantes.

El problema que yo veo a esta propuesta es que si la clase presencial consiste en aclarar las dudas de lo que se ha visto en casa (o simplemente fuera del aula o al margen de la presencia del profesor), lo más probable es que el tiempo dedicado a este aprendizaje autónomo sea muy superior al estrictamente presencial. Pongámonos en el caso de una clase de Matemáticas sobre las derivadas, como la ofrecida en este vídeo: https://www.youtube.com/watch?v=_6-zwdrqD3U. La explicación se extiende a lo largo de poco menos de cuarenta minutos, el tiempo que duraría una clase presencial. Sin embargo, con el vídeo es posible parar la explicación en cualquier momento o ir hacia atrás o tomar notas al ritmo de cada cual o, simplemente, verlo cuando se considere conveniente (eso sí, respetando los calendarios de cada asignatura). Esta es la razón por la que los familiares de Khan (2014) -el creador de la famosa academia online homónima- decían que le preferían en vídeo antes que en persona. Por otro lado, estar en clase implica que hay que entender las cosas y suministrar respuestas en un opresivo contexto de inmediatez.

\footnotetext{
Tal y como se puede ver en https://www.youtube.com/watch?v=tdG1Q6q1_w0.

4 Remito al lector interesado a esta entrada en mi blog: http://rfeito.blogspot.com/2019/06/un-espanol-en-italia.html.

5 Se puede ver una explicación en https://www.youtube.com/watch?v=UXK6gtyjICc.
} 
Muy posiblemente, los cuarenta minutos más arriba citados terminen por ser cien si tomamos en consideración las pausas y retrocesos. Una vez hecha esta labor, en la sesión siguiente los alumnos irían a clase a consultar sus dudas. Pero, ¿qué pasaría si la explicación en youtube está tan clara que apenas hay dudas o no hay ninguna? ¿Dejaríamos sin empleo a nuestro profesor de Matemáticas? Mi propuesta es que no. Pero está claro que una enseñanza de este tipo requiere redefinir radicalmente el rol del profesor. Con estos conocimientos adquiridos autónomamente sería posible analizar o investigar temas que, de un modo consensuado, se planteen desde el grupo de alumnos junto con su profesor. En definitiva, se trataría de ir más allá del mero aprendizaje de la mecánica de resolución de problemas y conectar el conocimiento científico con la realidad (por ejemplo, y dependiendo de la edad de los estudiantes, se podría trabajar con datos del Instituto Nacional de Estadística).

Pero aprender no consiste solo o fundamentalmente en escuchar o ver a un profesor en el aula o en Internet. Consiste también en leer ${ }^{6}$, investigar y, por qué no, memorizar lo que haya que memorizar. Me voy a otra materia. Por ejemplo, la Historia. Comprender la historia requiere, quizás más que ver vídeos, leer libros y artículos de revistas especializadas. ¿Cuánto tiempo se precisa para leer un libro -o unos libros- que posteriormente se pueda comentar en clase?

Una situación como la actual debería traducirse en un impulso a la lectura. ¿No debería un estudiante a partir de primero de la ESO -y quizás mucho antes- leer un mínimo de treinta o cuarenta libros a lo largo del año natural? Y, claro está, no me olvido de la creciente importancia de la lectura de la prensa generalista: no se puede ser plenamente un ciudadano si no se está informado y si no se desarrolla una interpretación fundamentada del mundo en el que se vive. Obviamente, no es lo mismo un lector de diez años que otro de dieciséis. Lo que pude comprobar en mi trabajo de campo en colegios de primaria es que hay muchos libros que no distinguen de edades de lectores. Por ejemplo, en un proyecto realizado en el colegio La Navata por niños de $4 .^{\circ}$ de primaria (de diez años de edad) trabajaron el tema del cine. Varios de los alumnos decidieron leer biografías de gentes del cine como, por ejemplo, Chaplin. ¿Qué edad es apropiada para leer un libro de este tipo? (Feito, 2020).

\section{Menos horas en el aula}

Hasta ahora estoy hablando de asignaturas. Sería perfectamente posible, tal y como hacen algunos centros, trabajar por proyectos (Feito, 2020). De esta manera, se rompe la separación entre materias al globalizar los contenidos curriculares. Más allá de esta posibilidad, también cabe la alternativa de introducir otras maneras de trabajar que formalmente son consideradas como asignaturas. Este sería el caso de la llamada monografía del Bachillerato Internacional: una investigación que ha de acometer el estudiante. En este mismo sentido, creo que es muy interesante el trabajo de investigación que tienen que realizar los estudiantes de bachiller de Cataluña. Se trata de un trabajo que todo alumno inicia en el primer curso de este nivel y que presenta tanto por escrito - un texto de entre 25 y 50 páginas- como oralmente. Este trabajo tiene la consideración de una asignatura más y le corresponden setenta horas de trabajo (poco menos de la mitad de tiempo de un universitario Trabajo de Fin de Grado de seis créditos). Se ha de realizar bajo la supervisión de un profesor del centro del alumno. Además, las universidades catalanas se ofrecen para colaborar con estos trabajos.

6 En francés se dice de una persona que está informada que «elle est à la page» (literalmente «está a la página»). El equivalente castellano es «estar al loro», es decir, tener capacidad de escucha. No caigamos en la tentación de hablar de «estar a la pantalla». 
Cuanto se ha dicho choca frontalmente con el excesivo número de horas de docencia de nuestro estudiantado de la ESO. Mientras que en España las horas lectivas de primaria (791) se encuentran en la media tanto de la OCDE (799) como de la UE22 (775), su número se dispara considerablemente en secundaria. En este nivel, en España son 1044, frente a una media de 915 en la OCDE y de 895 en la UE22. Son pocos los países en los que esta diferencia sea tan marcada. En el caso de España, las horas lectivas de secundaria son un 32\% más que las de primaria (OCDE, 2016). Tantas horas de clase en secundaria $-y$ quizás también en primaria- restringen la posibilidad de un trabajo autónomo.

Pero, quizás habría que ir más lejos. ¿Por qué se habría de ir todos los días a clase o tener tantas horas diarias de docencia? Lo que aquí se propone es reducir considerablemente el número de horas que el alumno pasa en clase. Si, por ejemplo, alguien tiene que hacer un trabajo sobre Zurbarán, sería aconsejable que dedicara unas cuantas jornadas a visitar el museo del Prado. De paso podríamos resolver el problema de hacer que los adolescentes se levanten mucho antes de lo que su biorritmo les indica para acudir a sus centros escolares y que convierte en improductivo buena parte del tiempo escolar de las primeras horas de la jornada.

Parte de estas propuestas requerirían cambiar la arquitectura de nuestros centros: necesitaríamos más espacios y más cómodos para poder leer (y de paso jamás castigar a los alumnos con permanecer confinados en la biblioteca del centro), salas para debatir en pequeños grupos, más laboratorios, más salas de música. Antes de diseñar un nuevo centro, se debería consultar con el profesorado y la comunidad educativa que vaya a trabajar allí.

\section{4. ¿̇Y la Universidad?}

Casi todos quienes escribimos en esta prestigiosa revista de sociología somos no solo investigadores, sino que también somos profesores. Así que de te fabula narratur. Lo que aquí he planteado se puede y se debe trasladar con mucha más radicalidad a la universidad. En mi opinión, tenemos demasiadas horas de clase presencial. Todo lo que sea mera transmisión de información se puede subir sin mayor problema a Internet. Es más, podríamos generar redes de profesores de una misma asignatura para, entre todos, crear vídeos o materiales de muy diverso tipo de cada uno de los temas de un programa compartido en mayor o menor grado. En este sentido, un trabajo como el acometido en solitario por Francesc Hernàndez podría ser un buen punto de partida ${ }^{7}$ En algunas facultades - como la mía: la de Sociología de la Complutense- contamos con un excelente laboratorio de investigación cualitativa con salas insonorizadas, cámaras, micros y demás que, a partir de ahora, podrían ser un excelente lugar para grabar las clases.

Una propuesta como esta exige un estudiante mucho más activo del que habitualmente tenemos -más bien, creamos- en la docencia presencial. Si cada cual ha visto previamente la lección y leído los textos pertinentes, la clase debe estructurarse en torno a las inquietudes, dudas, sugerencias, comentarios, etcétera que manifieste el estudiantado. De este modo, se acabaría con la idea de acudir a clase para recibir pasivamente mensajes a cargo del profesor.

Una de las cosas que más me preocupa de la existencia de asignaturas cuatrimestrales en los grados -frente a las anuales de las licenciaturas- es la de cuándo concedemos tiempo suficiente a nuestros estudiantes - pienso fundamentalmente en los de Sociología, pero creo que se podría generalizar a toda la uni-

\footnotetext{
Se puede ver en https://www.uv.es/fjhernan/curso/.
} 
versidad-para que lean libros enteros que puedan ser debatidos en clase. En esto, la sociedad civil hace tiempo que se ha anticipado con plataformas como meetup. Buena parte de las convocatorias amparadas por esta web consiste en reunir - habitualmente en un pub-durante un par de horas a personas que previamente han leído un libro -casi siempre una obra literaria-. Al igual que en el bachillerato de Cataluña, a lo mejor tendríamos que habilitar varios créditos - cada uno de ellos equivale a 30 horas de trabajo del estudiante- dedicados a este menester.

Buena parte de la actividad de los estudiantes consiste en realizar trabajos y me parece muy bien que así sea. En mi opinión, deberían llamarse más bien presentaciones. No debería bastar con el mero hecho de entregar al profesor un texto y obtener una calificación. Es posible que ese texto sea resultado de un encargo a una empresa o a otra person ${ }^{8}$. La única manera de saber -aunque sea aproximadamente- si el escrito es de otro -O, por lo menos, si se sabe de qué se habla- es recurrir a una presentación y defensa en público del escrito. Pero, y esto es lo más importante, con una presentación y defensa en público se desarrollan competencias de comunicación clave, tanto para el escenario productivo como para la construcción de una ciudadanía democrática.

\section{5. ¿Qué hacer una vez se vuelva a la docencia presencial?}

El 16 de abril de 2020, el diario New York Times ${ }^{9}$ publicaba un inquietante editorial sobre la posibilidad de que una amplia generación de estudiantes quedara marcada por la suspensión de la docencia presencial ${ }^{10}$. En él se mencionaban varios informes. Uno de ellos (NACSA, 2016) se refiere a la experiencia -en los EE.UU.- de escuelas charter (algo así como concertadas) de enseñanza virtual. La primera de ellas se creó a finales de los noventa, y en 2014 ya eran 135 (distribuidas entre 24 estados). El dato es contundente: en este tipo de escuelas (y para cualquier grupo demográfico, sea en función de la renta, la etnia o el hábitat) los resultados son claramente peores que en las presenciales. La organización que promueve este informe es netamente partidaria de las escuelas virtuales, ya que atienden a colectivos muy específicos y que difícilmente encontrarían otra alternativa escolar. Se trata de alumnos de zonas rurales que tratan de evitar un largo viaje en autobús hasta el colegio, de estudiantes-deportistas que precisan un horario flexible, de estudiantes hospitalizados y de alumnos de secundaria que encuentran aquí una segunda oportunidad. El problema que se detecta en este informe es que esta enseñanza virtual está diseñada para estudiantes que tengan una gran fuerza de voluntad - de hecho, los cursos se siguen al ritmo de cada cual- y que cuenten con una familia en condiciones de ayudarles. Por tanto, aquí tenemos una importante lección para el retorno a la escuela: hay que pensar detenidamente en cómo organizar la enseñanza virtual de modo que sea provechosa para todo tipo de alumnos.

Contamos ya con diversas experiencias de periodos prolongados en los que los alumnos no han podido recibir clase. Von Hippel (2020) citaba, entre otros, el caso de una huelga de profesores de más de dos meses de duración en Valonia (Bélgica) en 1990. Aquí se detectó que los alumnos afectados por tal conflicto tenían más probabilidades de repetir curso que el resto de sus compañeros. Sin embargo, señala Von Hippel, este no tendría que ser necesariamente el caso ahora, y ello porque hay un mínimo de dos elementos mitigadores. El primero es la tecnología. Si bien es cierto que no llega a todos los alumnos, sí alcanza a la mayoría. El segundo es la familia. A diferencia de lo que sucedía en el pasado, hoy hay un por-

\footnotetext{
8 Tal y como puede verse aquí: https://www.eldiario.es/cv/TFG_por_encargo-comprar_trabajo_de_fin_de_grado-comprar_TFG_0_970453707.html.

9 https://www.nytimes.com/2020/04/16/opinion/coronavirus-schools-closed.html.

10 Entre nosotros, Ismael Sanz (2020) apuntaba algo similar.
} 
centaje mucho más alto tanto de padres como de madres en el hogar-bien porque teletrabajan o porque simplemente no pueden acudir a sus puestos de trabajo-. No obstante, es sabido que con independencia de que cuenten o no con recursos tecnológicos, no todos los progenitores están capacitados para enseñar a sus retoños. Está claro, los hogares son más desiguales que la tecnología.

No sabemos si este prolongado periodo de confinamiento tendrá efectos similares al llamado «verano del olvido» (Heyns, 1978), término que se refiere a la pérdida de saberes escolares en septiembre con respecto a junio. El New York Times propone -y yo añadiría que especialmente para aquellos alumnos de menor nivel sociocultural y económico- clases durante varios veranos. Mi propuesta sería contar con voluntarios de todo tipo para impartir clases. El contenido de estas no tendría por qué coincidir completamente con el del currículo escolar. De hecho, se podría enseñar - entre otras muchas cosas- a redactar con coherencia, a hacer lecturas o experimentos poco habituales en el escenario escolar, a desarrollar destrezas artísticas. Se me ocurre que no sería un abuso buscar voluntarios entre los miles de altamente cualificados prejubilados que hay en España para que puedan ofrecer sus conocimientos durante quizás un par de semanas a comienzos de julio o a finales de agosto. Esta bolsa de voluntarios también la podrían conformar profesores y estudiantes de universidad ${ }^{11}$ (en la mayor parte de nuestras facultades hay aulas enormes que garantizarían la distancia social para evitar contagios). Y, claro está, también se podrían reclutar voluntarios entre los maestros y profesores, tanto en activo como jubilados.

\title{
6. Conclusiones
}

Una enseñanza con menos presencia del profesor y menos localizada en al aula es toda una invitación a la autonomía de las personas, a su capacidad para organizar sus tiempos. En una memorable conferencia en la Residencia de Estudiantes de Madrid en 1930, Keynes hablaba de que al cabo de cien años los avances tecnológicos serían tales que bastaría con trabajar quince horas a la semana. Y esto es lo que señalaba el economista inglés:

\begin{abstract}
Asi es que por primera vez desde su creación el hombre se enfrentará a su verdadero y permanente problema: cómo usar su libertad sin la presión de las preocupaciones económicas, cómo ocupar el ocio, que la ciencia y el interés compuesto le habrán concedido, para vivir sabia, agradablemente y bien. (Keynes, 1931: 121).
\end{abstract}

Ojalá se cumpliesen las previsiones de Keynes y tuviéramos tanto tiempo libre. En todo caso, vivimos más años como jubilados que nunca. Quizás uno de los problemas más graves del confinamiento sea justamente este: ¿qué hacer con este tiempo disponible? De nuestra experiencia escolar deberíamos aprender a dedicar tiempo diario a la lectura (y no solo de textos literarios), a la conversación inteligente (habitual en el buen lector), a conocer los avances científicos y tecnológicos, al disfrute y práctica de las artes (creo que todo el mundo debería ser capaz de tocar un instrumento musical o de pintar), al cuidado de los demás y de nuestro medio ambiente, a la práctica del deporte y seguro que me dejo en el tintero multitud de cosas. En definitiva, lo que aquí planteo es que la escuela debería ser clave en la creación de una sociedad de personas libres y solidarias, y esta podría ser la lección fundamental de esta crisis.

11 La Universidad Complutense ha promovido que sus estudiantes de Matemáticas puedan impartir clases virtuales a los hijos de su comunidad universitaria. 


\section{Referencias bibliográficas}

Bergmann, Jonathan y Sams, Aaron (2014). Flipped Learning. Gateway to Student Engagement. International Society for Technology and Education.

Feito, Rafael (2020). ¿Qué hace una escuela como tú en un siglo como este? Madrid: La Catarata.

Fórum Aragón (2020): “Especial cuarentena por la COVID-19”, Fórum Aragón, 29.

Heyns, Barbara (1978). Summer Learning and the Effects of Schooling. Nueva York: Academic Press.

Keynes, John Maynard (1931). Essays in Persuasion. Londres: Macmillan.

Khan, Salman (2014). The One World Schoolhouse: Education Reimagined. Nueva York: Hachette.

NACSA (2016): "A Call to Action to Improve the Quality of Full-Time Virtual Charter Public Schools". Disponible en https://www.publiccharters.org/sites/default/files/migrated/wp-content/ uploads/2016/06/Virtuals-FINAL-06202016-1.pdf.

OCDE (2016). Panorama de la educación. Indicadores de la educación 2016. Madrid: Instituto Nacional de la Calidad Educativa. Recuperado de: http://www.mecd.gob.es/dctm/inee/eag/panorama2016okkk. pdf?documentId=0901e72b82236f2b.

Sanz, Ismael (2020): “Efectos de la crisis del coronavirus sobre la educación”, Magisterio, 19 de marzo.

Von Hippel, Paul T. (2020): “How Will the Coronavirus Crisis Affect Children's Learning? Unequally. It's not a vacation. There's still half a semester of curriculum to learn”. Education Next, 20 de abril. https:/ / www.educationnext.org/how-will-coronavirus-crisis-affect-childrens-learning-unequally-covid-19/\#.

\section{Nota biográfica}

Rafael Feito es catedrático de Sociología en la Universidad Complutense de Madrid. Es autor de Nacidos para perder. Un análisis sociológico del rechazo y del abandono escolares (Madrid, CIDE, 1990), Estructura social contemporánea. Las clases sociales en los países industrializados (Madrid, Siglo XXI, 1995), Clases sociales y comportamiento político en España (Madrid, Entinema, 1998), Los retos de la educación obligatoria (Barcelona, Ariel, 2000), Una educación de calidad para todos. Reforma y contrarreforma educativas en la España actual (Madrid, Siglo XXI, 2002), Otra escuela es posible (Madrid, Siglo XXI, 2006), Construyendo escuelas democráticas (compilador junto con J. I. López, Hipatia, Barcelona, 2008), Los retos de la participación escolar (Madrid, Morata, 2011), ¿Qué hace una escuela como tú en un siglo como este? (Madrid, La Catarata, 2020) y de numerosos artículos en revistas especializadas y generalistas. 